\title{
Structure Optimization of Solute Molecules via Free Energy Gradient Method
}

\author{
Masataka Nagaoka \\ Graduate School of Human Informatics, Nagova Universitv, Chikusa-ku, Nagova +64-8601, Japan \\ Recewed March 5, 2003
}

\begin{abstract}
Fundamental ideas of the free energy gradient method are briefly reviewed with three applications: the stable stnictures of glycine and ammonia-water molecule pair in aqueous solution and the transition state (TS) stnucture of a Menshutkin reaction $\mathrm{NH}_{3}+\mathrm{CH}_{3} \mathrm{Cl} \rightarrow \mathrm{CH}_{3} \mathrm{NH}_{3}{ }^{+}+\mathrm{Cl}^{-}$in aqueous solution, which is the first example of full TS optimization of all internal degrees of freedom
\end{abstract}

Key Words : Free energy' gradient. Stnicture optimization. Transition state

\section{Introduction}

In a lot of chemical, biological and environmental phenomena. the chemical reaction dynamics in solution or across interfoce plays a very important role. where the microscopic solvation structures of solute molecules offer essential and inevitable information. ${ }^{1}$ To obtain a stationary point on a multi-dimensional free energy surface (FES), ${ }^{1}$ e.g., stable state (SS) or transition state (TS) in solution chemical reaction. a new TS optimization method, the free energy gradient (FEG) method, has been developed. ${ }^{2-9}$ Analogous to the method for the BonOppenheimer ( $\mathrm{BO}$ ) potential energy surface using ab initio molecular orbital (MO) calculation. the FEG method utilizes force and hessian on the FES, which can be calculated by molecular dynamics (MD) method and the free energy perturbation (FEP) theory. ${ }^{11.11}$ Furthermore. on the basis of the method. we have proposed the definition of the intrinsic reaction coordinate (IRC) on the $\mathrm{FES}^{+}$

In this review article, the theoretical idea of FEG method is first introduced in the next section (Theoretical Treatments) together with the algorithmic procedure of structure optimization. Next in the section (Application). three applications are summarized: first for the glvcine in water. second for the anmonia-water molecule pair in water. and third for the TS optimization of a Menshutkin reaction $\mathrm{NH}_{3}+\mathrm{CH}_{3} \mathrm{Cl} \rightarrow \mathrm{CH}_{3} \mathrm{NH}_{3}^{+}+\mathrm{Cl}^{-}$in aqueous solution, which is the first example of full TS optinization on the multidimensional FES.

\section{Theoretical Treatments}

A. Structure Optimization. In MD sinulation, forces acting on each atom of the solute molecule by all solvent molecules are always calculated every time step. By timeaveraging these forces, the "force" on FES, i.e., a minus of FEG, is obtained as a function of the solute molecular structure $\mathbf{q}^{\mathrm{s}}$ as follows, ${ }^{4.7 .9}$

$$
\mathbf{F}^{\mathrm{FE}}\left(\mathbf{q}^{\mathrm{s}}\right)=-\frac{\partial G\left(\mathbf{q}^{\mathrm{s}}\right)}{\partial \mathbf{q}^{\mathrm{s}}}=-\left\langle\frac{\partial V_{\mathrm{RS}}\left(\mathbf{q}^{\mathrm{s}}\right)}{\partial \mathbf{q}^{\mathrm{s}}}\right\rangle .
$$

Tel\&Fax: +81-52-789-5623; http://frontier ncube.human.nagova-u. ac.jp/E-mail: mnagaokaa $\widehat{a}$ info.human.nagoya-u.ac.jp where $G\left(\mathbf{q}^{s}\right)$ is the FE function and $V_{\mathrm{RS}}\left(\mathbf{q}^{\mathbf{s}}\right)$ is the sum of the solute intramolecular potential energy and the solute-solvent interaction energy at $\mathbf{q}^{s}$. The brackets $\langle\ldots\rangle$ in Eq. (1) denote the time average that is equal to the equilibrium ensemble average.

$$
\langle\ldots\rangle=\frac{\int d \mathbf{q}^{\mathrm{B}}(\ldots) \exp (-\beta V)}{\int d \mathbf{q}^{\mathrm{B}} \exp (-\beta V)} .
$$

where $V$ is the whole potential of solution, under the condition that the solute molecular structure is restricted to be $\boldsymbol{q}^{s}$ and $\boldsymbol{q}^{\mathrm{E}}$ denotes the solvent coordinates as a whole.

For structure optimization. in the FEG method with the steepest-descent-path following procedure, the $i+$ lth reactant structure $\mathbf{q}_{i+1}^{s}$ is taken to be

$$
\mathbf{q}_{i+1}^{s}=\mathbf{q}_{i}^{s}+\Delta \mathbf{q}_{i}^{s} .
$$

where an adaptive displacement vector $\Delta \mathbf{q}_{i}^{\varsigma}$ is defined as

$$
\Delta \mathbf{q}_{i}^{\mathrm{s}} \equiv \mathcal{c}_{i} \cdot \mathbf{M}^{-1} \cdot \mathbf{F}_{i}^{\mathrm{FE}}
$$

by multiplying

$$
\mathbf{F}_{i}^{\mathrm{FE}} \equiv \mathbf{F}^{\mathrm{FE}}\left(\mathbf{q}_{i}^{\mathrm{s}}\right)=-\left\langle\frac{\partial V_{\mathrm{RS}}\left(\mathbf{q}^{\mathrm{s}}\right)}{\partial \mathbf{q}^{\mathrm{s}}}\right\rangle,
$$

by an adaptive constant $c_{i}$ of dimension $\mathrm{T}^{2}$ and the inverse of the mass matrix.

$$
\mathbf{M}=\left[\begin{array}{lllllll}
m_{1} & & & & & \\
& m_{1} & & & & & \\
& & m_{1} & & & \\
& & & \ddots & & \\
& & & m_{N} & & \\
& & & & & \\
& & & & & & \\
& & & & & m_{N}
\end{array}\right]
$$

where $m_{3}$ is the $j$ th atomic mass of $N$-atomic molecule involved

To obtain the stable molecular structure in solution, the 
procedure in the FEG method is summarized as the following processes:

[P1] Start with the geometry $\mathbf{q}_{k}^{\mathrm{s}}, k=0$.

[P2] For $\mathbf{q}_{k}^{\mathrm{s}}$. calculate the force on the FES $\mathbf{F}_{k}^{\mathrm{FE}}$

[P3] Find the stationary point using the force:

$$
\Delta \mathbf{q}_{k}^{\mathrm{s}}=c_{k} \cdot \mathbf{M}^{-1} \cdot \mathbf{F}_{k}^{\mathrm{FE}}
$$

If the force $\mathbf{F}_{k}^{\mathrm{FE}}$ is small enough within the tolerance of convergence and/or the predicted change in the geometry $\Delta \mathbf{q}_{k}^{\mathrm{s}}$ is small enough to be satisfied with the condition

$$
\left\langle\frac{\partial V_{\mathrm{RS}}\left(\mathbf{q}^{*}\right)}{\partial \mathbf{q}^{\mathrm{s}}}\right\rangle=0 \text { : zero-gradient condition. }
$$

then. stop. setting the step number $n_{\text {tin }}$.

[P4] Set $\mathbf{q}_{k+1}^{\mathrm{s}}=\mathbf{q}_{k}^{\mathrm{s}}+\Delta \mathbf{q}_{k}^{\mathrm{s}}, k=k+1$ and return to $\mathrm{P} 2$.

B. Free Energy Optimization. During the structure optimization procedure as the solute molecular geometry $\mathbf{q}^{s}$ varies from $q^{s}$ to $q_{j-1}^{s}$ (Eq. (3)), the corresponding FE change $\Delta G_{i}$ is provided with by the FEP theory ${ }^{10.11}$ and is obtained by

$$
\begin{aligned}
\Delta G_{i} & =G_{j-1}-G_{j} \\
& =-k_{\mathrm{B}} T \ln \left\langle\exp \left[-\beta\left\{V_{\mathrm{RS}}\left(\mathbf{q}_{i-1}^{\mathrm{s}}\right)-V_{\mathrm{RS}}^{\prime}\left(\mathbf{q}_{i}^{\mathrm{s}}\right)\right\}\right]\right\rangle_{j},
\end{aligned}
$$

where the subscript $i$ in the average $\langle\ldots\rangle_{i}$ means that it is taken over the sampling at $q^{s}$. Thus. the FE profile can be drawn by connecting these differences from the initial structure $\mathbf{q}_{13}^{s}$ to the final one $\mathbf{q}_{\mathrm{spt}}^{\mathrm{s}}$ at optimization. Then. the FE change of stabilization $\Delta G_{\text {stabl }}$ results in

$$
\Delta G_{\text {stals }}=\sum_{i=0}^{n_{\text {fin }}} \Delta G_{j} \text {. }
$$

\section{Application}

A. Glycine in Water. First the FEG method was applied to the multidimensional geometry optimization of glycine zwitterion $(\mathrm{ZW})$ in aqueous solution in order not only to demonstrate its applicability but also to examine its efficiency. The solute potential energy $V_{R S}\left(q^{s}\right)$. i.e. the sum

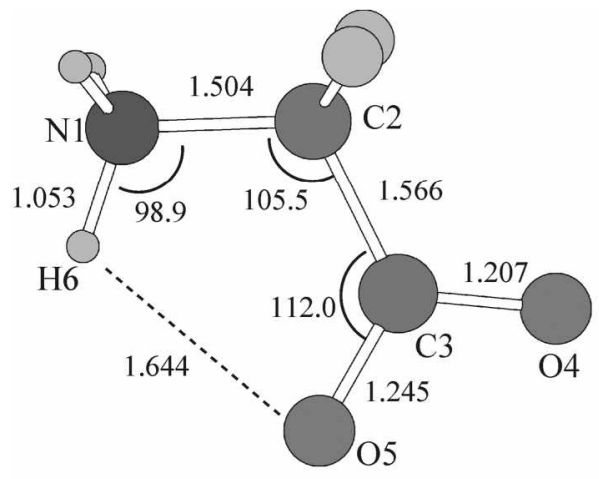

(a) GAS phase of the $\mathrm{ZW}$ intramolecular potential energy and the interaction between $\mathrm{ZW}$ and a solvent water molecule (TIP4P) was described by a fitted potential energy function obtained previously by the empirical valence bond (EVB) method. ${ }^{5.612-14}$ Then, the most stable $\mathrm{ZW}$ structure in aqueous solution was obtained within the tolerance assumed. and it was found that the FE and enthalpy changes of stabilization from the initial geometry optimized in the gas phase are -0.9 and $-3.5 \mathrm{kcal} / \mathrm{mol}$. respectively. and the amino and carbosyl groups are spatially separated each other due to their solvating with water molecules (Figure 1). ${ }^{7}$ Comparing the contributions of enthalpy and entropy to FE. the former is attributed to the main origin of the FE stabilization during the optimization procedure. and it was found that the solvation entropy prevents water molecules from solvating the $\mathrm{ZW}$ more strongly.

B. $\mathrm{NH}_{3}+\mathbf{H}_{2} \mathbf{O} \rightarrow \mathrm{NH}_{4}^{+}+\mathbf{O H}^{-}$. Second, corresponding to the reactant state of the ionization reaction $\mathrm{NH}_{3}+\mathrm{H}_{2} \mathrm{O} \rightarrow$ $\mathrm{NH}_{4}^{+}+\mathrm{OH}^{-}\left(K_{a}=2.24 \times 10^{-5}\left(25^{\circ} \mathrm{C}\right)(\right.$ Expt. $\left.)\right)$. the hydrated structure of ammonia molecule in aqueous solution was theoretically optimized as an ammonia-water molecule pair $\left(\mathrm{H}_{3} \mathrm{~N} \cdots \mathrm{H}_{2} \mathrm{O}\right)$ by the FEG method (Figure 2). The $\mathrm{H}_{3} \mathrm{~N} \cdots$ $\mathrm{H}_{2} \mathrm{O}$ potential energy. i.e., the sum of the intrapair potential energy and the interaction between the pair and a solvent water molecule (TIP3P) was described by a hybrid quantum mechanical and molecular mechanical (QM/MM) method combined with a semiempirical MO method at the PM3 level of theory. ${ }^{9}$ In the QM/MM formalism, $V_{\mathrm{RS}}\left(\mathbf{q}^{\mathrm{s}}\right)$ can be represented. for an instantaneous solvent configuration $q^{B}$, as

$$
V_{\mathrm{RS}}\left(\mathbf{q}^{\mathrm{s}}\right)=\left\langle\Psi\left|\hat{H}_{\mathrm{QMI}}+\hat{H}_{\mathrm{QN} / \wedge \mathrm{N}}\right| \Psi\right\rangle,
$$

where $\Psi$ denotes the SCF wave function of electrons of the solute molecules $\left(\mathbf{q}^{5}\right)$ in solution. In Figure 3. both $\Delta G(300$ $\mathrm{K})$ and $\Delta V_{R}$ are shown as functions of the step number, which denote the $\mathrm{FE}$ change at $300 \mathrm{~K}$ and the corresponding change in the solute potential energy $V_{\mathrm{R}}\left(=\left\langle\Psi\left|\hat{H}_{\mathrm{QM}}\right| \Psi\right\rangle\right)$. respectively. At the first step of optimization. $\Delta G$ decreases suddenly to almost $-0.3 \mathrm{kcal} / \mathrm{mol}$, while it becomes almost flat up to 9. Presently. judging from the calculated root mean square values. ${ }^{9}$ the geometry at the step number 8 is taken to be the most stable structure of the ammonia-water molecule

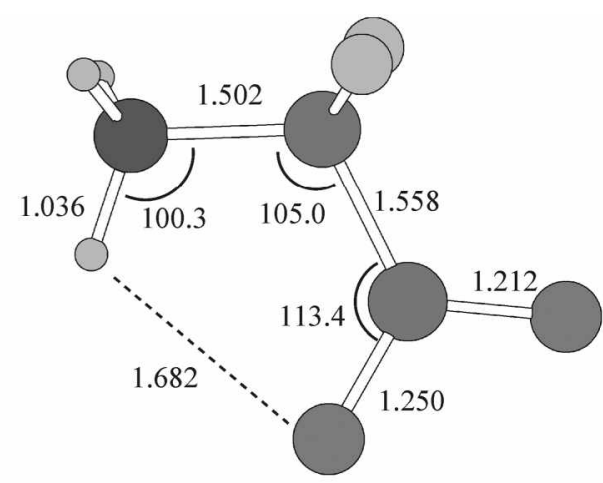

(b) FEG Method

Figure 1. Stable-state structures optinized (a) in the gas phase by the HF level of theory and (b) in the aqueous solution obtained by the FEG method. Bond lengths are in $\AA$ and bond angles are in degree. 


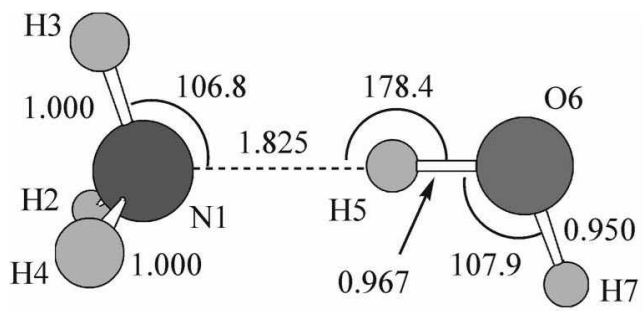

(a) GAS phase (PM3)

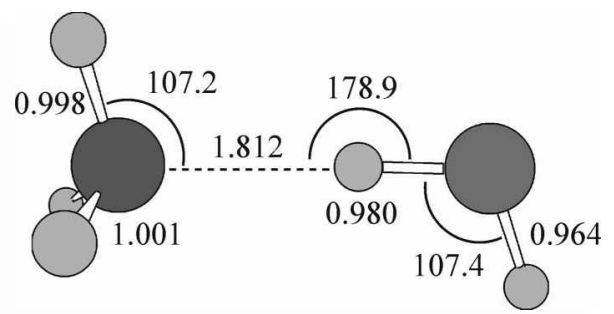

(b) FEG Method (8th step)

Figure 2. Stable-state structures optunized (a) in the gas phase by the PM3 and (b) in the aqueous solution obtained by the FEG method. Bond lengths are in $\AA$ and bond angles are in degree.

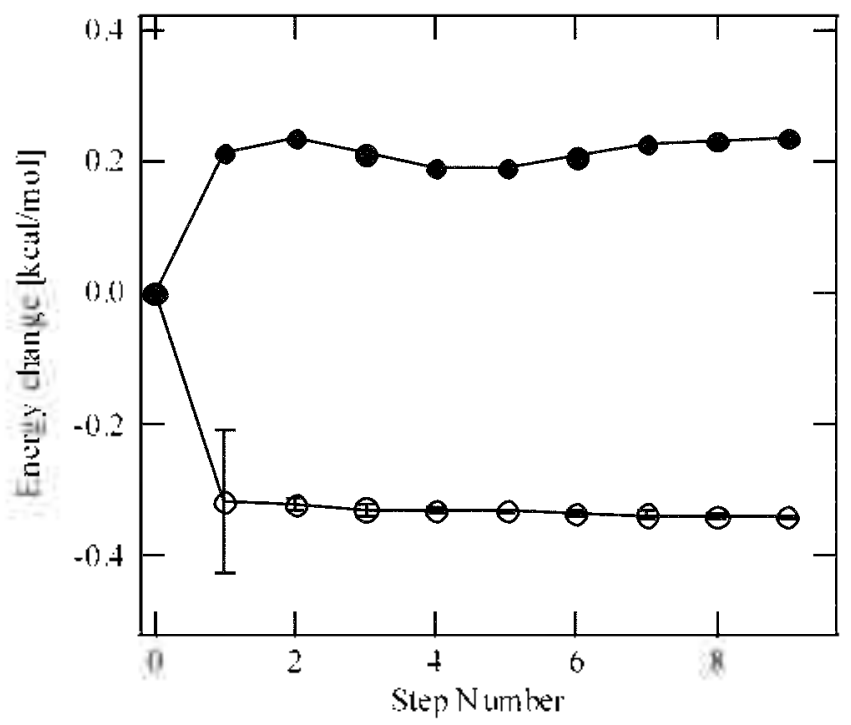

Figure 3. Free energy change (open curcles with an enor bar) and solute potential energy change (closed circles) of the stabilization of ammonia-water $I: 1$ pair in aqueous solution using the FEG method conbined with the QMMM-MD method at the PMs level of theory.

pair, and then the FE change of stabilization was estimated $-0.3 \mathrm{kcal} / \mathrm{mol}$ from the $\mathrm{FE}$ for the same structure as that of the cluster in gas phase.

The optimized structure was found to be almost the same as that in the gas phase except a longer $\mathrm{OH}$ bond length of the water molecule (Figure 2). However. its realization in solution is accomplished by virtue of subtle fulfillment of both "zero gradient" condition (Eq. (8)) and "force balance" condition:

$$
\left\langle\frac{\partial\left\langle\Psi\left|H_{\mathrm{QM}}\right| \Psi\right\rangle}{\partial \mathrm{q}^{\mathrm{s}}}\right\rangle=-\left\langle\frac{\partial\left\langle\Psi\left|\hat{H}_{\mathrm{QM} / \mathrm{M}}\right| \Psi\right\rangle}{\partial \mathrm{q}^{\mathrm{s}}}\right\rangle
$$

meaning the compensation for the balance between the solute intramolecular potential energy gradient and the forces acting on each solute atom due to solvation. ${ }^{9}$

C. $\mathrm{NH}_{3}+\mathrm{CH}_{3} \mathrm{Cl} \rightarrow \mathrm{CH}_{3} \mathrm{NH}_{3}^{+}+\mathrm{Cl}^{-}$-.-The First Example of Full TS-optimization. Recently. the TS for the Menshutkin reaction $\mathrm{NH}_{3}+\mathrm{CH}_{3} \mathrm{Cl} \rightarrow \mathrm{CH}_{3} \mathrm{NH}_{3}^{+}+\mathrm{Cl}^{-}$in aqueous solution was located on the FES with respect to all internal degrees of freedon of the reactant molecules (Figure 4) ${ }^{8}$ As explained in the subsection III-B, the solutesolvent system was described by a lyybrid QM/MM method. ${ }^{8}$ The TS thus obtained (Figure $4(b)$ ) was found earlier than that in the gas phase (Figure 4(a)), which is in accord with the Hammond postulate. As a result, the reaction path in water was found to deviate largely from that in the gas phase. " One can understand therefore that, in aqueous solution. the reaction should proceed accompanied with such a structural deformation to enluance the charge separation. It is concluded that, in such a reaction including charge separation. TS structure optimization on an FES is inevitable for obtaining valid information about a TS in polar solution. ${ }^{8}$

Finally, it is concluded that the present FEG method works quite well in spite of its simple optimization scheme equipped with only the adaptive displacement vector.

Acknowledgements. The author thanks Prof. K. S. Kin for his kindly inviting me to contribute to the special issue of Bulletin of Korean Chemical Society for the Proceedings of the loth Korea-Japan joint symposium. This work was supported partly by a Grant-in-Aid for Science Research

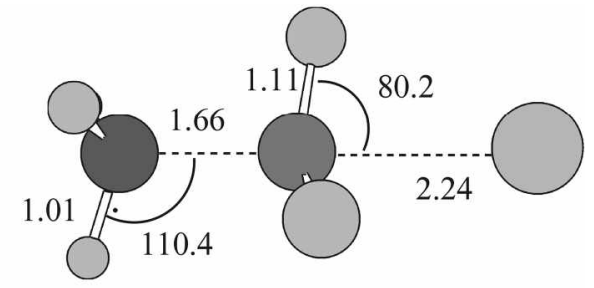

(b) FEG Method

Figure 4. Transition-state structures optimized (a) in the gas phase by the AMI and (b) in aqueous solution obtained by the FEG method. Bond lengths are in $A$ and bond angles are in degree. 
from the Ministry of Education. Science and Culture in Japan.

\section{References}

1. Computer Hodeling of Chentical Reactions in Enzwmes and Solutions: Warshel, A.: Wiley: New York. 1991

2. Nagaoka, M: Okuno, Y: Yoshida. N.: Yamabe, T. Int J. Quant. Chem. 1994. 51.519-527.

3. Nagaoka. M: Okuno. Y: Yoshida. N.: Yamabe. T. In The Proceeding of the Third China-Japa Symposimm on Theoretical Chemistry Xiamen, 1994: 31

4. Oklyama-Yoshida. N.: Nagaoka, M.: Yamabe. T. Int. J. Otantum Chem. 1998, 70.95-103.

5. Nagaoka. M.: Okuyama-Yoshida. N.: Yamabe. T. J. Phns. Chem.
A $1998,102.8202-8208$

6. Okuyama-Yoshida. N.: Nagaoka. M.: Yamabe. T. ibid. 1998. 102. 285-292

7. Okuyama-Yoshida. N.: Kataoka. K.: Nagaoka. M.: Yamabe. T. $J$. Chem. Phus. 2000. 113. 3519-3524.

8. Hirao. H. Nagae. Y: Nagaoka. M. Chem. Phns. Lett 2001. $3+8$. $350-356$

9. Nagae. Y: Ohishi. Y.; Naruse, N.; Nagaoka, M. subntitted for publication.

10. Zwarzig. R. W. J. Chem. Plys. 1954. 22. 1420-1426.

11. Singh. U. C.: Brown. F. K.: Bash. P. A.: Kollman. P. A. J. Am. Chent Soc. 1987, 109. 1607-1604.

12. Warshel. A.; Weiss, R. M. J. An. Chem. Soc. 1980, 102, 62186226.

13. Aqvist. J.: Warshel. A. Chem Rev 1993. 93.2523-2544.

14. Chang. Y.-T.: Miller. W. H. J. Plys. Chem 1990. 94. 5884-5888. 